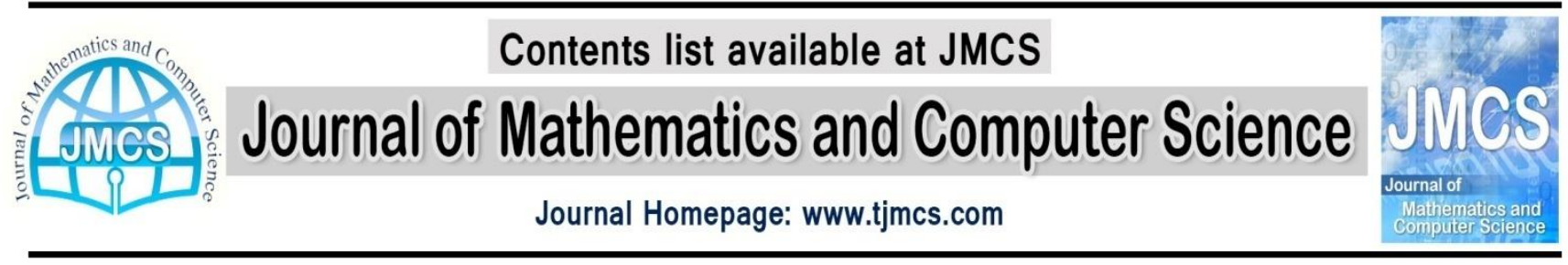

\title{
Optimizing of Spatial Domain Watermark Recovery Algorithm by Laplacian Filter
}

\author{
Mehdi Alirezanejad ${ }^{1}$, Saeed Amirgholipour ${ }^{2}$, Vahid Saffari ${ }^{3}$, Moein Arab $^{4}$, Shahin Aslani ${ }^{4}$ \\ ${ }^{1}$ Department of Computer, Firuzkooh Branch, Islamic Azad University, Firuzkooh, Iran. \\ ${ }^{2}$ Department of Computer, Ramsar Branch, Islamic Azad University, Ramsar, I. R. Iran \\ ${ }^{3}$ Department of Computer, Shiraz Branch, Islamic Azad University, Shiraz, I. R. Iran \\ ${ }^{4}$ Department of Computer, Sharif University of technology international Campuse, Kish, I. R. Iran.
}

E-mail:m_arn2004@yahoo.com,s.kasmani@iauramsar.ac.ir,saffari@iaushiraz.ac.ir,arab, sh_aslani@kish.sharif.edu

Article history:

Received August 2014

Accepted September 2014

Available online October 2014

\begin{abstract}
In this paper, impact of a Laplacian filter in better watermark recovery in the spatial domain watermarking algorithms has been investigated. A Laplacian filter is an enhancement filter, this filter could be utilized to optimize extraction of the watermark information in spatial domain watermarking. The distinction between the watermark and unwatermarked part is increased by this filter. Thus, watermark could be extracted significantly better by the watermark recovery algorithm. To show effectiveness of Laplacian filter on the spatial domain watermarking algorithms, it is applied on a typical correlation based method. Several experiments are done to show that performance of correlation based watermarking algorithm is improved by applying this filter before watermark extraction procedure.
\end{abstract}

Keywords: Laplacian Filter, Digital Image Watermarking, Correlation Based Watermarking, Spatial Domain Watermarking.

\section{Introduction}

In recent years, Digital watermarking is one of the most important techniques for the copy right protection. In this techniques, some extra information in the form of watermark is embedded invisibly in the digital media [1-2].

In the design of the watermarking algorithm always, there are two important item that they should be considered: robustness and imperceptibility. The imperceptibility means that how much the embedded information make reductions in signal quality; and the robustness is the ability of the watermark to remain readable after innocent or malicious signal processing operations on the watermarked image. These parameters have conflict with each other [3]. 
The Watermarking methods could be categorized into the spatial domain or the transform domain. The watermark is embedded directly in the pixels of the host image in the spatial domain techniques. Although, these methods are not robust to image common image processing operation [4-5], they have high imperceptibility. Some methods [6-7], utilized the filters capability to better extract the watermark. Transform domain watermarking schemes benefit properties of the transform domain to embed the watermark. These methods usually use the Discrete Cosine Transform (DCT) [8-9] and the Discrete Wavelet Transform (DWT) [10-11]. These methods typically are more robustness to image manipulations.

Several methods are proposed to augment the performance of algorithms watermarking. Most of these method concentrate on finding most suitable place for embedding watermark. Human visual system (HVS) [12-13], just noticeable difference (JND) [14-15] and optimal quantization matrix are used as techniques for finding most proper place [16]. These methods are commonly utilizing the characteristics of in frequency domain to detect suitable place. On the other hand, these method need some extra time to process digital media. Thus, they are not suitable for the real time application [17].

However, small research are done to augment the watermark recovery algorithm. The purpose of these method are to boost watermark detection rate by an efficient and simple model. Consequently, these approaches could increase the resistance and the imperceptibility of watermarking algorithms. On the other hand, these methods could apply and used in real time.

The watermark information is considered as noise for the watermarked image in its spatial domain. This noise is magnified before detection, and then recovers the watermark information by adjusting the extracted data from the frequency domain according to the global minimum method [18]. A preprocessing method is proposed that exploit a combination of Laplacian and Laplacian of Gaussian filtering to improve recovering the watermark from watermarked image in the DCT-based watermarking algorithm $[3,17]$. They prove that preforming these filter before watermark recovery in the DCT based watermarking could significantly increase chance of watermark recovery. Application of High Boost and unsharp masking filters in boosting the detection of watermark in show in $[5,7]$. They show that preforming this filter before and in the middle of watermark extraction procedure could significantly augment the results.

In this paper, a Laplacian filter is applied before watermark extraction to increase the probability of detection of watermark in the correlation based watermark recovery algorithms in the spatial domain methods. This filter could assistance the recovery algorithm to discriminate between the watermark and the host image. Thus, the watermark information could be removed from the altered watermarked image significantly better. This filter is applied before executing watermark extraction procedures in the spatial domain watermarking method. Different Experiments are done to show the impact of applying Laplacian filter in the spatial domain based watermarking.

\section{MATERIALS}

\subsection{Second-order headings}

Laplacian filter is one of the sharpening filters. The principal objective of sharpening filter is to highlight and enhance detail in the image that has been blurred. Uses of image sharpening to vary and include applications ranging from electronic printing and medical imaging to industrial inspection and autonomous guidance in military systems. 
Laplacian filter could obtained by first and second derivative. In most applications, the second derivative is better suited than the first derivative for image enhancement because of the ability of the former to enhance fine detail. For this, and for reasons of simpler implementation and extensions, in this paper, the second derivative is used as enhancement.

In this paper, Gonzales definition is used for Laplacian filter [19]. It can be shown that the simplest isotropic derivative operator is the Laplacian, which, for a function (image) $f(x, y)$ of two variables, is defined as.

$\nabla^{2} f=\frac{\nabla^{2} f}{\partial^{2} x^{2}}+\frac{\nabla^{2} f}{\partial^{2} y^{2}}$

Because derivatives of any order are linear operations, the Laplacian is a linear operator. In order to be useful for digital image processing, this equation needs to be expressed in the discrete form. There are several ways to define a digital Laplacian using neighborhoods. Taking into account that we now have two variables, we use the following notation for the partial second-order derivative in the x-direction:

$\frac{\nabla^{2} f}{\partial^{2} x^{2}}=f(x+1, y)+f(x-1, y)-2 f(x, y)$

and, similarly in the $y$-direction, as

$\frac{\nabla^{2} f}{\partial^{2} y^{2}}=f(x, y+1)+f(x, y-1)-2 f(x, y)$

The digital implementation of the two-dimensional Laplacian in equation (4) is obtained by summing these two components:

$$
\nabla^{2} f=[f(x+1, y)+f(x-1, y)+f(x, y+1)+f(x, y-1)]-4 f(x, y)
$$

This equation can be implemented using the mask shown in equation (5).

$$
\left[\begin{array}{ccc}
0 & -1 & 0 \\
-1 & 4 & -1 \\
0 & -1 & 0
\end{array}\right]
$$

In this paper a special implementations of the Laplacian is used:

$$
\nabla^{2} f=8 f(x, y)-\left[\begin{array}{l}
f(x-1, y-1)+f(x-1, y)+f(x-1, y+1)+f(x, y-1) \\
+f(x, y+1)+f(x+1, y-1)+f(x+1, y)+f(x+1, y+1)
\end{array}\right]
$$

This equation can be implemented using the mask shown in equation (7).

$$
\left[\begin{array}{ccc}
-1 & -1 & -1 \\
-1 & 8 & -1 \\
-1 & -1 & -1
\end{array}\right]
$$


Because the Laplacian is a derivative operator, its use highlights gray-level discontinuities in an image and deemphasizes regions with slowly changing gray levels. Background features can be "recovered" while still preserving the sharpening effect of the Laplacian operation simply by adding the original and Laplacian images.

\subsection{Motivation of Laplacian filtering}

The goals of this research were to show the effectiveness of preforming typical Laplacian filtering on performance of spatial domain watermarking method. This filter is applied on attacked watermarked image before execution of the watermark extraction algorithm. This filter process applies a special kind of edge enhancement operations, which separate watermarked and un-watermarked parts of image. This filter helps that watermark information, which is different from the image background, becomes recognizable to the watermark extraction procedure.

\section{METHODS}

In this paper, a typical spatial domain watermarking method is used [7]. It is correlation based watermarking using block processing in the spatial domain.

\subsection{Watermark Embedding Algorithm}

The watermark embedding process is represented in Figure 1., followed by a detailed explanation.

Step 1: Divide the host image into $16 \times 16$ blocks.

Step 2: Re-formulate the watermark image into a vector of zeroes and ones.

Step 3: Generate two uncorrelated pseudorandom sequences by a key. One sequence is used to embed the watermark bit 0 (PN_0) and the other sequence is used to embed the watermark bit 1 (PN_1). Number of elements in each of the two pseudorandom sequences must be equal to the number of block.

Step 4: Embed the two pseudorandom sequences, PN_0 and PN_1, with a gain factor $\alpha$ in the $16 \times 16$ blocks of the host image. If we donate $X$ as the matrix of the block, then embedding is done as equation (8):

$$
X^{\prime}= \begin{cases}X+\alpha^{*} P N 0 & \text { watermark_bit }=0 \\ X+\alpha^{*} P N 1 & \text { watermark_bit }=1\end{cases}
$$

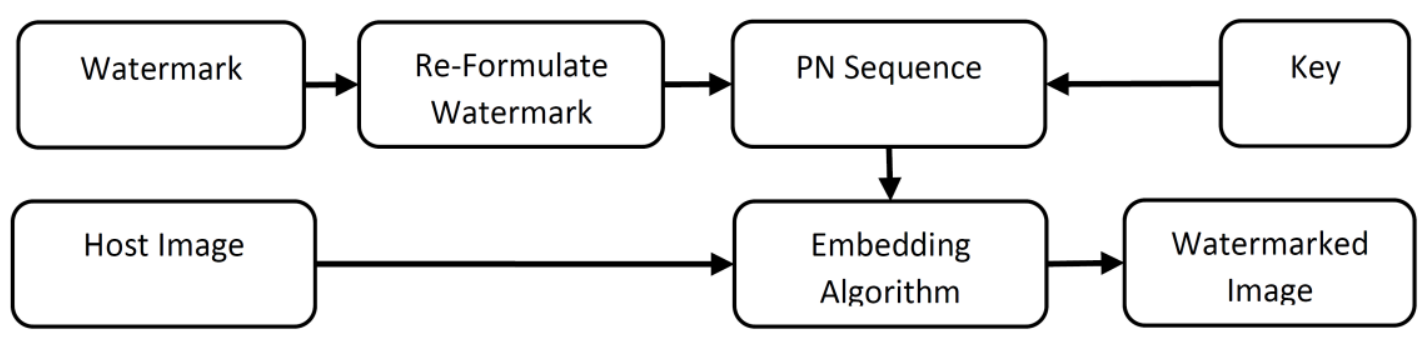

Figure 1. The watermark embedding process 


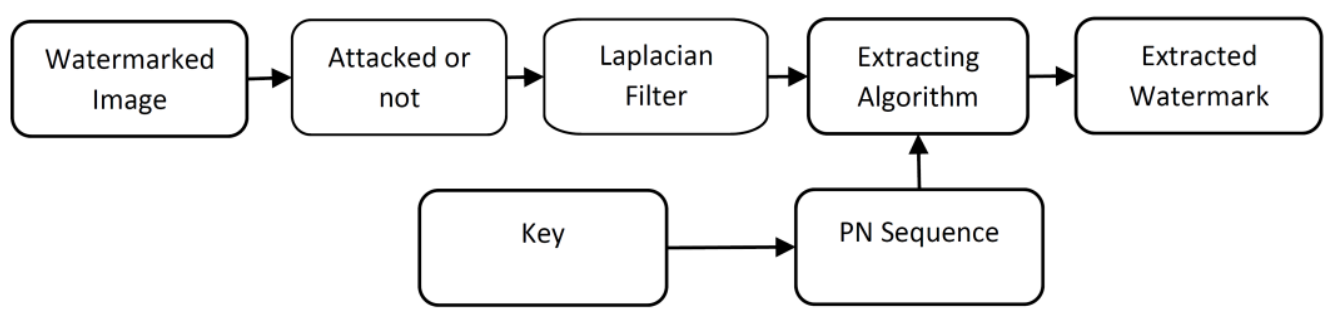

Figure 2. The watermark extracting with applying Laplacian before preforming extracting algorithm.

\subsection{Watermark Extracting Procedure}

The typical correlation based algorithm is a blind watermarking algorithm, and thus the original host image is not required to extract the watermark. Extraction algorithm is the same as embedding one, and filtering is used before applying it to better separate watermark information from host image. The watermark extraction procedure is shown in Fig. 2, and described in details in the following steps.

Step 1: applying proposed filter as shown in the equation of (2) for Laplacian filter, on the watermarked image.

Step 2: Divide watermarked image that could be attacked or not into $16 \times 16$ blocks.

Step 3: Regenerate the two pseudorandom sequences (PN_0 and PN_1) using the same key which used in the watermark embedding procedure.

Step 4: For each block in the watermarked image calculate the correlation between the element and the two generated pseudorandom sequences (PN_0 and PN_1). If the correlation with the PN_0 was higher than the correlation with PN_1, then the extracted watermark bit is considered 0 , otherwise the extracted watermark is considered 1.

Step 5: The scrambled watermark is reconstructed using the extracted watermark bits.

\section{Experimental Results}

To compare the efficiency of the proposed filter on correlation based methods, three standard grayscale images with different contents of size $512 \times 512$ are used in our experiments, as shown in Figure 3. (a)-(b). Pepper is used as a representation of image with low spatial frequency and Barbara as a representation of image with average spatial frequency. In this experiment, a $64 \times 64$ binary image, as shown in Figure 3.(c) is taken as the watermark of images. The impact of the Laplacian filter is investigated by measuring imperceptible and robustness of watermarked image. For the imperceptible capability, a quantitative index, Peak Signal-to-Noise Ratio (PSNR), is employed to evaluate the difference between an original image $O$ and a watermarked image. For the robust capability, the Mean Absolute Error (MAE) measures the difference between an original watermark $W$ and the corresponding extracted one. If a method has lower MAE, it is more robust. The PSNR and the MAE are, respectively, defined by equation 9 and 10; respectively, 


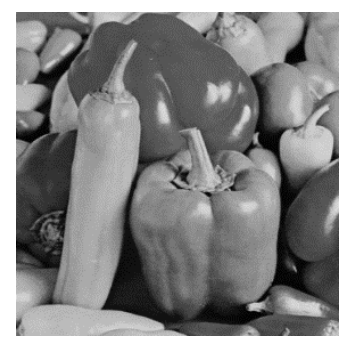

(a)

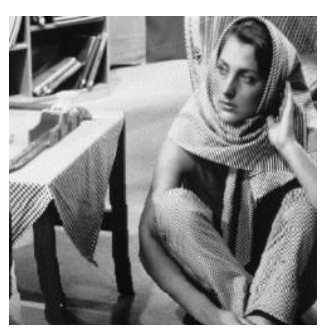

(b)

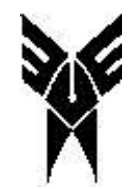

(c)

Figure. 3. (a) The original Pepper image; (b) the original Barbara image; (c) the original watermark.

$$
\operatorname{PSNR}(\mathrm{O}, \overline{\mathrm{O}})=10 \log \frac{255 \times 255}{10 \frac{\sum_{\mathrm{i}=0 \mathrm{j}=0}^{\mathrm{I}-1 \mathrm{~J}-1}\left(\left\|\mathrm{O}_{\mathrm{ij}}-\overline{\mathrm{O}}_{\mathrm{ij}}\right\|\right)^{2}}{\mathrm{I} \times \mathrm{J}}}
$$

$\operatorname{MAE}(W, \hat{W})=\frac{\sum_{i=0}^{S-1}\left\|w_{i}-\hat{w}_{i}\right\|_{1}}{|W|}$

Where \|\|$_{1}$ and $|\cdot|$ stand for the L1 norm, and the number of components of a vector, respectively.

Performance of the proposed method is compared to previous method [6]. The algorithm was tested on Peppers and Barbara standard test images. The watermarked image $\mathrm{O}$ is obtained following the completion of the watermark embedding procedure. The watermark information is embedded with PSNR 30, 35, and $40 \mathrm{~dB}$ in the watermarked images. Then Laplacian method which is described in Section 2 is performed on these watermarked images which may be attached. This filtered image is used as an input of extraction procedure. MAE between the original $\mathrm{W}$ and the extracted watermark $W^{\prime}$ is calculated for different PSNRs. The performance of the proposed method is compared with Depovere method and with the default results when no processing is done on the correlation based watermarking algorithm. In the Depovere method, for each block in the watermarked image, at first a high boost filter is applied on them and then, correlation between the filtered block and the two generated pseudorandom sequences ( $\mathrm{PN} \_0$ and $\mathrm{PN} \_1$ ) is computed. To show the robustness of these methods, the efficacy of the algorithm is tested by several attacks, including JPEG compression, image scaling, adding Salt and Pepper noise, Gaussian filtering. The presented method is implemented using MATLAB.

\subsection{Comparison in the case of Attack Free}

In the first experiment, we compared the effect of Laplacian filter on watermarked image, which is not attacked, with Depovere's method and normal extracted watermark (without filtering). The results are shown in form of a line chart in the Figure 4 . The results show significant improvement in compare with the normal one. Although, the results show that the Depovere method is performed as similar as the proposed method. 

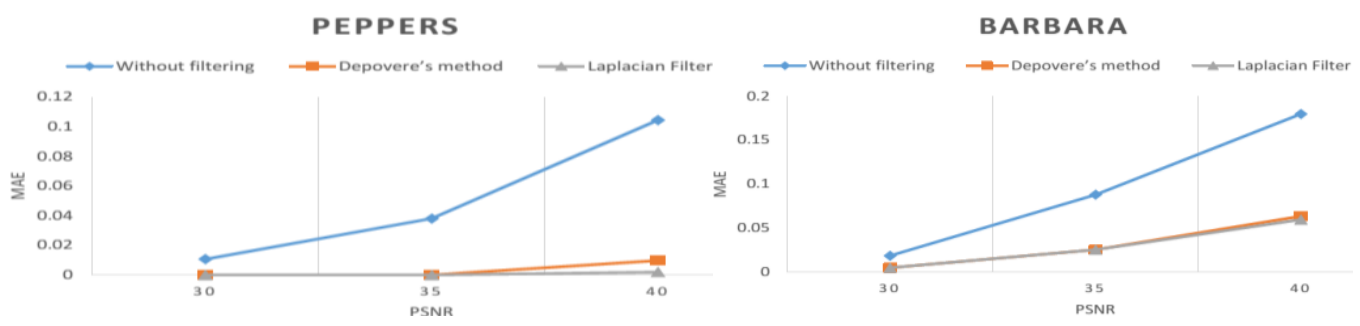

Figure 4. The experimental results in terms of the MAE in which watermarked images did not manipulated by the attacks in correlation
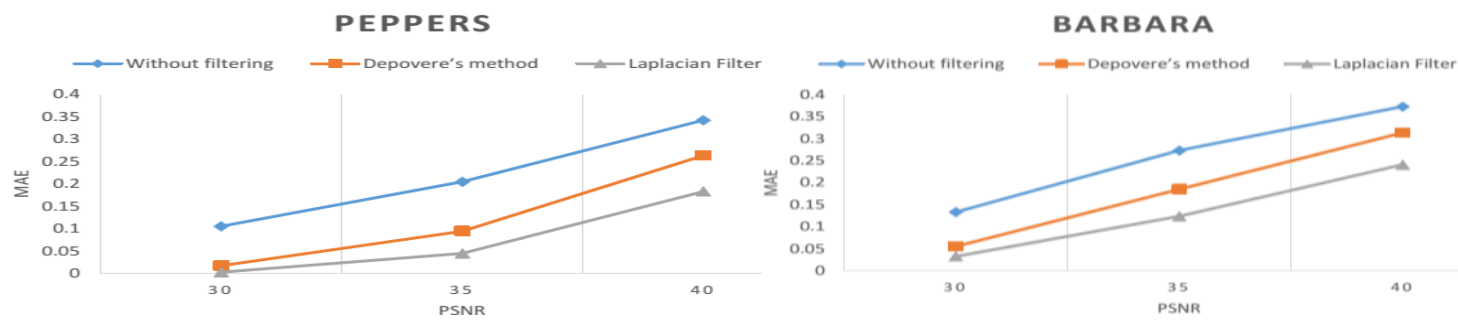

Figure 5. The experimental results in terms of the MAE in which watermarked images further manipulated by the $50 \%$ JPEG compression attack on correlation based method.
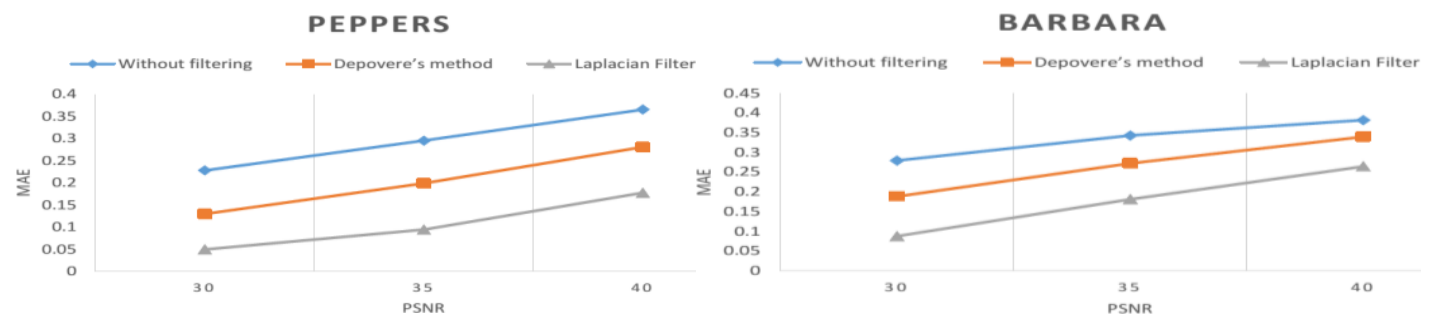

Figure 6. The experimental results in terms of the MAE in which watermarked images further manipulated by the $50 \%$ scaling attack on correlation based method.

\subsection{Comparison in the Case of JPEG Compression Attack}

In the second experiment, we wanted to find out how the proposed algorithm improves the robustness of correlation based method when $50 \%$ jpeg compression attack is applied to the watermarked image. The results achieved from executing Laplacian filtering, and Depovere method are shown in the Figure 5. As shown in this figure, although, the watermark retrieval performance is improved by Depovere method, but these results are become better by the proposed filter. Generally, it is believed, if the watermarked image has a higher PSNR, the watermark embedded into the image is more imperceptible. This figure also shows that, the watermark is recovered with more accuracy in compare with the previous method when the watermark is embedded in the host image with high PSNR.

\subsection{Comparison in the Case of Scaling Attack}

In the third experiment, we wanted to find out how much the proposed algorithm improves the robustness of correlation based method when the watermarked image is attacked by $50 \%$. The results achieved from executing Laplacian filtering, and Depovere method are shown in the Figure 6. As shown in this Figure, although, the watermark retrieval performance is improved by Depovere 
method, but these results are become well by the proposed filter. This Figure also shows that, the proposed method has a best performance in compare with the previous methods when the watermark is embedded in the host image with high PSNR.

\subsection{Comparison in the case Salt and Peppers Noise Attack}

In the fourth experiment, we wanted to find out how the proposed algorithm improves the robustness of correlation based method when 10\% Salt and Pepper noise attack is applied to the watermarked image. The results achieved from executing Laplacian filtering, and Depovere method are shown in the Figure 7. As shown in this figure, although, the watermark retrieval performance is improved by Depovere method, but these results are become better by the proposed filter. This Figure also shows that, the watermark is recovered with more accuracy in compare with the Depovere method when the watermark is embedded in the host image with higher PSNR.

\subsection{Comparison in the case Blurring Attack}

In the fifth experiment, we try to find out how the proposed algorithm improves the robustness of correlation based method when Gaussian Blurring (with $\mathrm{h}=5$, and $\sigma=1$ ) attack is applied to the watermarked image. The results achieved from Laplacian filtering, and Depovere method are shown in the Figure 8. In this case, the proposed method significantly shows more improvement at the watermark recovery. This figure also shows that, the watermark is recovered with more accurateness in compare with the Depovere method when the watermark is embedded in the host image with higher PSNR.
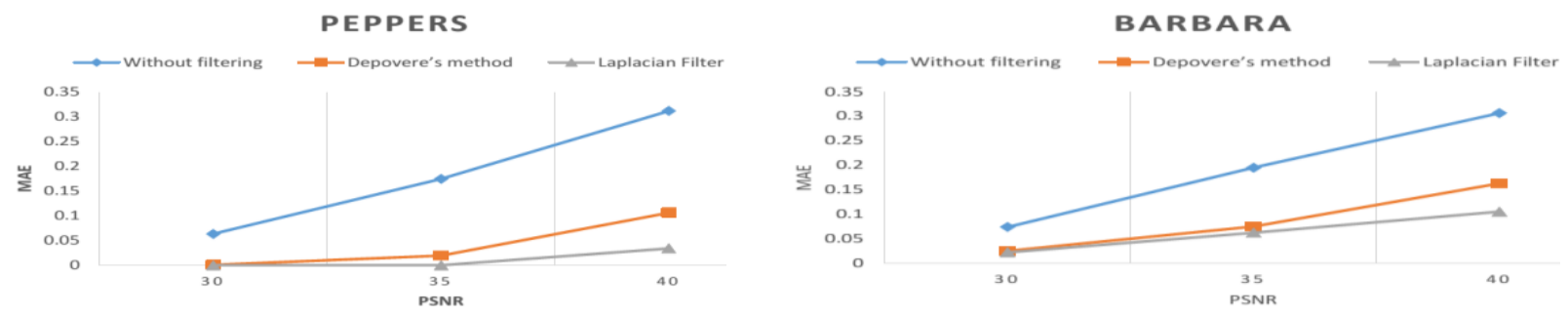

Figure 7. The experimental results in terms of the MAE in which watermarked images further manipulated by $10 \%$ salt and pepper attack on correlation based method.
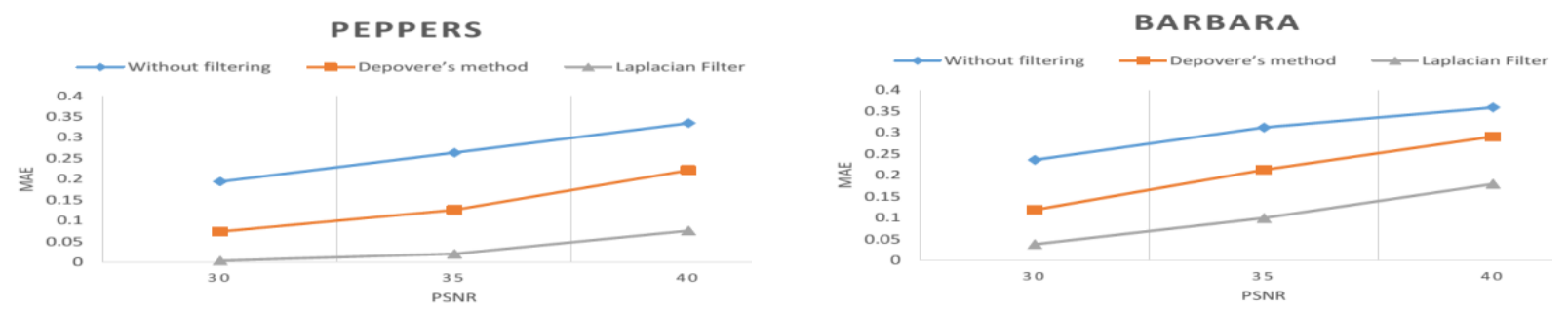

Figure 8. The experimental results in terms of the MAE in which watermarked images further manipulated by Gaussian Blurring with $\mathrm{h}=5$, and $\sigma=1$ attack on correlation based method.

\section{Conclusions}

In this paper, a new application of Laplacian filter is presented to achieve more imperceptibility and robustness in the spatial domain based watermarking methods. The dissimilarity between watermarked part and unwatermarked part of host image is increased by Laplacian filter. Then, this enhanced watermarked image is used as an input image watermark extraction's process. The proposed method helps details of watermarked image become perceptible. Several experiments are 
done to show that presented filtering method could considerably raise power of spatial domain watermarking method. Effectiveness of the proposed filtering method is tested by comparing its result with another filtering method, which proposed by Depovere's, in the term of MAE. The watermark is extracted after common image processing attacks with lower MAE value. Especially in case of enhancement operations with filtering and noise addition, increasing in performance is become more noticeable.

\section{ACKNOWLEDGEMENTS}

This paper is extracted from research projects for Islamic Azad University, Firuzkooh Branch, Iran.

\section{References}

1. Kasmani, Saied Amirgholipour, and Ahmadreza Naghsh-Nilchi, "A new robust digital image watermarking technique based on joint DWT-DCT transformation." In Convergence and Hybrid Information Technology, 2008. ICCIT'08. Third International Conference on, vol. 2, (2008), 539-544. IEEE.

2. Amirgholipour, Saeed K., and Ahmad Reza Naghsh-Nilchi., "Robust Digital Image Watermarking Based on Joint DWT-DCT." JDCTA 3, No. 2, (2009), 42-54.

3. Kasmani, Saied Amirgholipour, Meysam Mahfouzi, and Mohsen Asfia, "A New Pre-processing Approach to Improve DCT-Based Watermarkings Extraction." In Computer Science and Information Technology-Spring Conference, 2009. IACSITSC'09. International Association of, (2009), 131-135. IEEE.

4. Potdar, Vidyasagar M., Song Han, and Elizabeth Chang, "A survey of digital image watermarking techniques." In Industrial Informatics, 2005. INDIN'05. 2005 3rd IEEE International Conference on, (2005), 709-716. IEEE,

5. Saeed Amirgholipour,Ahmad Reza Naghsh-Nilchi,Aboosaleh Mohammad Sharifi, Mehdi Alirezanejad, Moien Arab "Application of Unsharp Mask in Augmenting the Quality of Extracted Watermark in Spatial Domain Watermarking", The Journal of Mathematics and Computer Science (JMCS), Vol 11, No. 2, (2014), 137-146.

6. Depovere, Geert, Ton Kalker, and J-P. Linnartz, "Improved watermark detection reliability using filtering before correlation." In Image Processing, 1998. ICIP 98. Proceedings. 1998 International Conference on, vol. 1, (1998), 430-434. IEEE.

7. Alirezanejad, M., Saffari, V., Amirgholipour, S., \& Sharifi, A. M. "Effect of Locations of Using High Boost Filtering on the Watermark Recovery in Spatial Domain Watermarking". Indian Journal of Science and Technology, Vol 7, No. 4, (2014), 517-524.

8. Chu, Wai C., 2003 "DCT-based image watermarking using subsampling." Multimedia, IEEE Transactions on 5, no. 1, (2003), 34-38.

9. Lin, Shinfeng D., and Chin-Feng Chen., "A robust DCT-based watermarking for copyright protection." Consumer Electronics, IEEE Transactions on 46, no. 3, (2000), 415-421,

10. Lu, W., Sun, W., \& Lu, H.," Robust watermarking based on DWT and nonnegative matrix factorization". Computers \& Electrical Engineering, 35(1), (2009), 183-188.

11. Sun, G., \& Yu, Y. (2007, May). DWT based watermarking algorithm of color images. In Industrial Electronics and Applications, 2007. ICIEA 2007. 2nd IEEE Conference on, (2007), 1823-1826. IEEE. 
12. Kutter, Martin, and Stefan Winkler, "A vision-based masking model for spread-spectrum image watermarking." Image Processing, IEEE Transactions on 11, no. 1, (2002), 16-25.

13. Levický, Dušan, and Peter Foriš., "Human visual system models in digital image watermarking." Radioengineering 13, no. 4, (2004), 38-43

14. Nguyen, P. B., Beghdadi, A., \& Luong, M., "Robust watermarking in DoG scale space using a multiscale JND model". In Advances in Multimedia Information Processing-PCM, Springer Berlin Heidelberg, (2009), 561-573.

15. Bouchakour, M., Jeannic, G., \& Autrusseau, F, "JND mask adaptation for wavelet domain watermarking". In Multimedia and Expo, 2008 IEEE International Conference on, (2008), 201-204.

16. Li, Q., \& Cox, I. J. "Using perceptual models to improve fidelity and provide resistance to valumetric scaling for quantization index modulation watermarking", Information Forensics and Security, IEEE Transactions on, 2(2), (2007), 127-139.

17. Arnirgholipour, S., and Sharifi, A. "A Pre-Filtering Method to Improve Watermark Detection Rate in DCT Based Watermarking". International Arab Journal of Information Technology (IAJIT), Vol 11, No.2, (2014), 178-185.

18. Pan, Zhigeng, Li Li, Mingmin Zhang, and David Zhang. , "Watermark extraction by magnifying noise and applying global minimum decoder." In Image and Graphics, 2004. Proceedings. Third International Conference on, (2004), 349-352. IEEE,

19. Gonzalez, Rafael C., and Richard E. Woods. "Digital Image Processing: Introduction." Upper Saddle River, NJ; 2002. 